\title{
Design and implementation of new family fire prevention and alarm
}

\author{
Xieyongzhi, ${ }^{1, a, 2, b}$, Huangchengzhi ${ }^{2, b}$ \\ ${ }^{1}$ Guangxi key laboratory of new energy and building energy saving, Guilin 541004, China \\ ${ }^{2}$ College of Mechanical and Control Engineering, Guilin University of Technology, Guilin 541004, \\ China
}

Keywords: single chip microcomputer; smoke sensor; alarm; infrared sensor; wireless.

Abstract: this paper designs a fire prevention and burglar alarm based on sensors and single chip microcomputer as the core components. First of all, a host computer and a slave computer are designed and made. The intelligent wireless alarm system is composed of a signal acquisition unit, a signal transmission unit and a control unit. It has theft-proof and fireproof functions, including stolensentiment detection by concealed installation, and facilitate multi-user unified management of the pyroelectric infrared sensor to complete, fire detection by the MQ-2 smoke sensor and temperature detector as a composite. The host and slave communicate information wirelessly so as to realize remote real-time monitoring.

\section{Introduction}

In recent years, with the rapid development of society and economy, modern buildings have large scale, high standards, large numbers of personnel and large numbers of equipment. Commercial networks, residential areas and high-rise buildings are increasing day by day, thus burying many hidden dangers in people's work and life, and fire hazard is one of them. With the acceleration of the process of urbanization in rural areas, the structure of urban residents is diverse, the social security situation has become complex and diverse, and accidental invasion threatens people's personal safety at all times. Social development, people's fire prevention, anti-theft awareness is constantly increasing, the urgent need for better monitoring and early warning devices to complete the fire, theft of early detection, early reporting, early preparedness, in order to reduce unnecessary losses. Therefore, people need a new type of fire prevention and theft prevention system.

\section{Overall design of the system}

Fire alarm system includes electronic detection and intelligent control, forming anti-theft, fire alarm system. The system consists of four main modules: burglar detector, fire detector, automatic alarm for client and wireless transmission. The system uses STC89C52 microcontroller as the core of control. It consists of infrared human body sensor, smoke sensor, temperature sensor, LED control circuit and relevant control management software. Realize remote monitoring and sound and light alarm function, sound and light alarm function, smoke detection, human detection and other functions. The overall block diagram of the system is shown in figure 1:

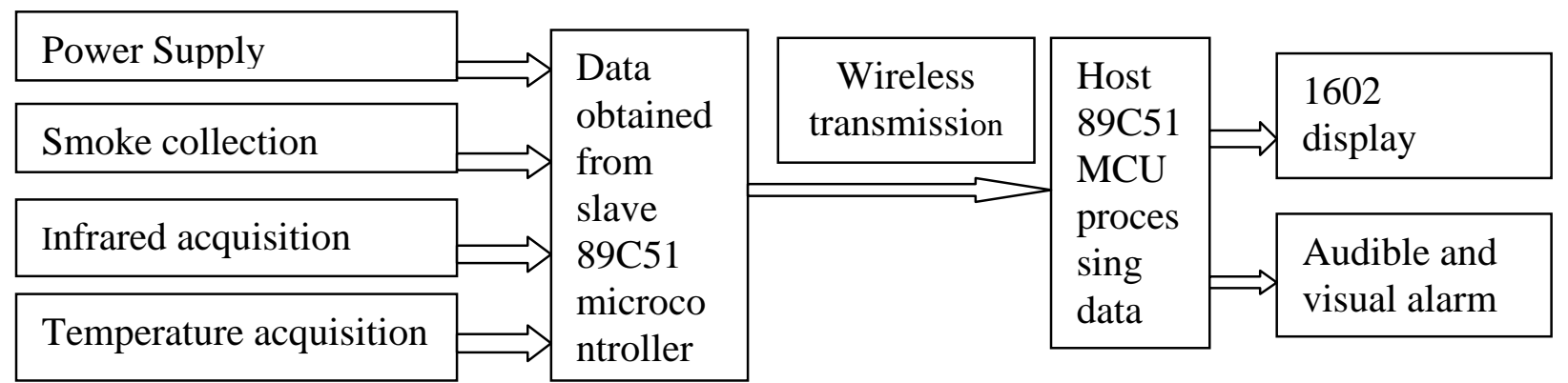

Figure 1 overall block diagram of the system 


\section{System hardware circuit design}

\section{Smoke detection circuit design}

Smoke sensing uses a MQ-2 sensor. Various smoke concentration signals are converted into voltage values through a smoke sensor, and the ADC0832 chip converts the voltage values into data which can be processed by the single chip, so that the smoke intensity alarming value can be determined in actual conditions. ADC0832 is a 8 bit resolution, dual channel A/D conversion chip produced by National Semiconductor Corporation Ns. Because of its small size, high cost performance and good compatibility, it is widely used by SCM enthusiasts and enterprises. The wiring diagram of the A/D conversion chip and sensor is shown in figure 2 :

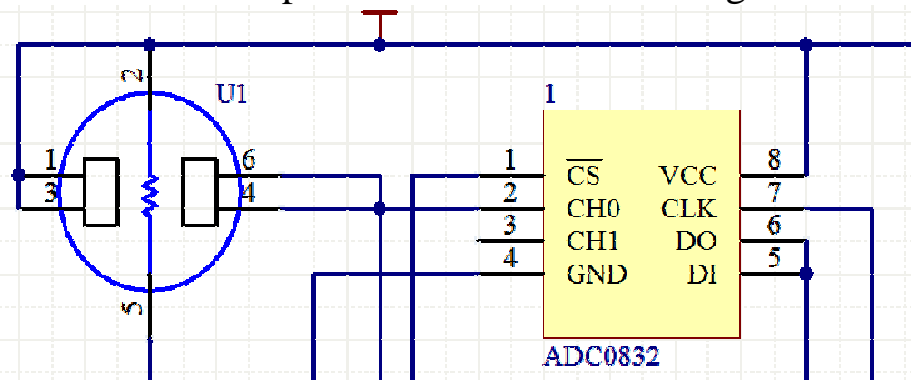

Figure 2 smoke concentration acquisition circuit

\section{Infrared signal acquisition circuit design}

The circuit converts the infrared radiation of the human body into electrical signals (as shown in Figure 3). Adjust the R13 according to different distance, and the maximum can be adjusted to 7 meters. Figure BISS0001 pin 1 pick in the high level, in the infrared sensor delay time, if the body has been in its sensing range, output will always keep a high level, only one left, from the high level into a low level. The circuit works in a trigger mode.

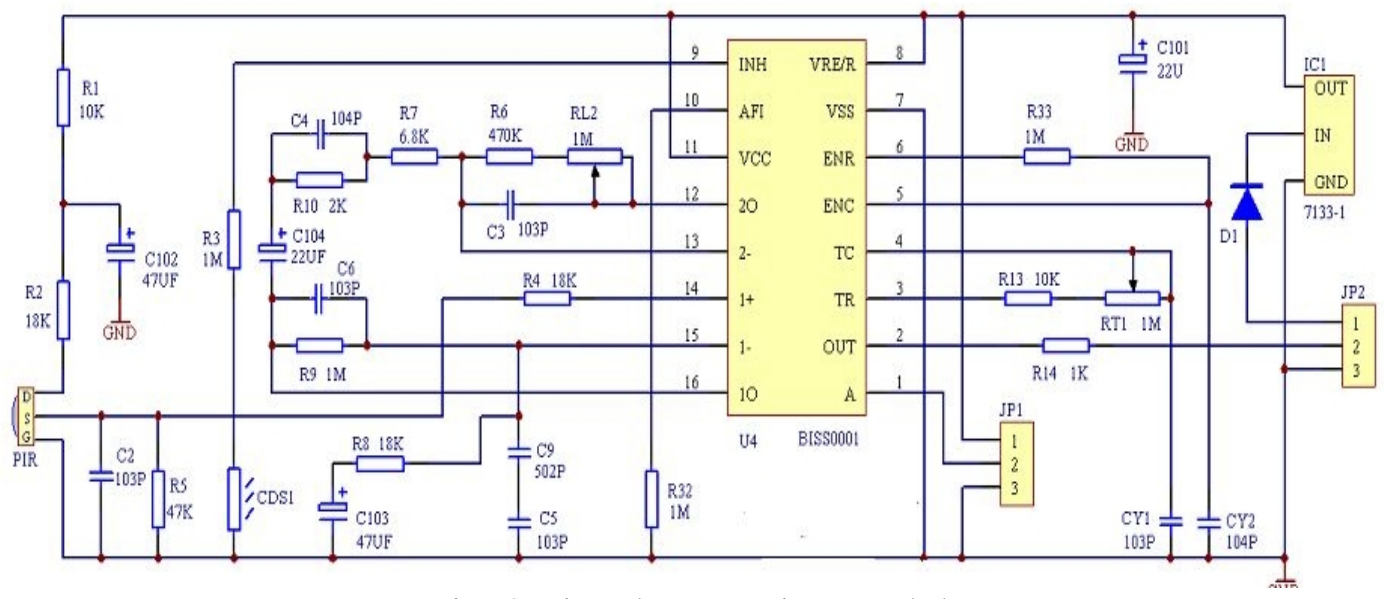

Fig. 3 signal processing module

\section{Sound and light alarm circuit design}

The sound and light alarm circuit is connected with a $2 \mathrm{~K}$ resistor through the base of the 9012 triode, and is connected with the P3.6 port of the singlechip. The information received by each module is transmitted to the microcontroller, and the upper limit is set by the software, and whether or not the value is higher than the upper limit is raised, and the sound and light alarm is issued when the value is higher than that of the singlechip. Buzzer and LED lighting wiring diagram as shown in figure 4: 


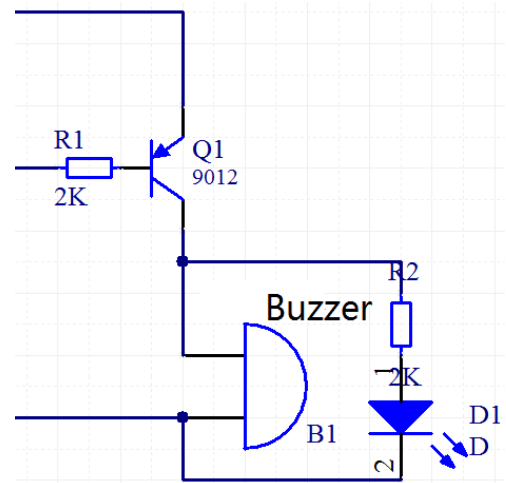

Figure 4 sound and light alarm circuit

\section{Design of wireless transceiver circuit}

The pin functions of the nRF24L01 are shown in figure 5:

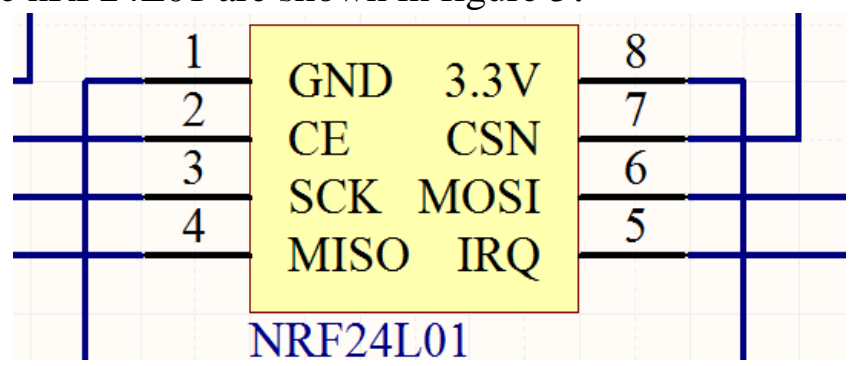

1.GND: ground terminal;

Figure 5 nRF24L01 package diagram

2.CE: Chip mode control line. In the case of low CSN, the CE cooperates with the CONFIG register of the NRF24L01 to determine the state of the NRF24L01;

3.SCK: chip-controlled clock line (SPI clock);

4.MISO: chip control data line (Master input, slave output)

5.MOSI: chip control data line (Master output, slave input)

6.CSN: chip select line chip, CSN is low when the chip works;

7.IRQ: Interrupt signal pin. When the interrupt occurs internally, the IRQ pin changes from high level to low level when an internal interrupt occurs on the NRF24L01 [27]. The pin will go low in the following three cases: TxFIFO is finished and an ACK is received (in the case of ACK), RxFIFO receives the data and reaches the maximum number of retransmissions;

8.3.3V: Input power 3.3v.

\section{System software design and Proteus simulation}

System workflow is: The slave first has to preheat the sensor because the mq-2 gas sensor must be warmed up for a few minutes before it can be used to avoid false positives. After the program is initialized, the slave will continue to transmit data. If the host receives the data, the red light will blink and the system will enter the monitoring state.

When the alarm system is working, the information obtained by the smoke concentration sensor is processed by the ADC0832 and then transmitted into the slave microcontroller. Temperature and infrared information is directly passed to the slave microcontroller processing, the processed data transmitted to the host MCU by wireless transmission module, The host microcomputer receives the information from the computer and analyzes it to determine whether the system should send an alarm. The host program also includes the use of LCD1602 display smoke concentration, emergency, manual alarm, alarm setting, various interrupt routines, etc..

In Proteus ISIS, the hardware is connected according to the schematic diagram. As the Proteus software does not have nRF24L01 device, can not carry out wireless transmission simulation, the 
original design of the schematic can not be input, it is necessary to merge the host and the slave, to remove nRF24L01 wireless transmission module. Connect each test module which is connected on the slave microcontroller to the host computer directly. Proteus simulation is mainly in order to write the program, you can simulate the operation of the program to check the problem. The Proteus simulation is shown in figure 6:

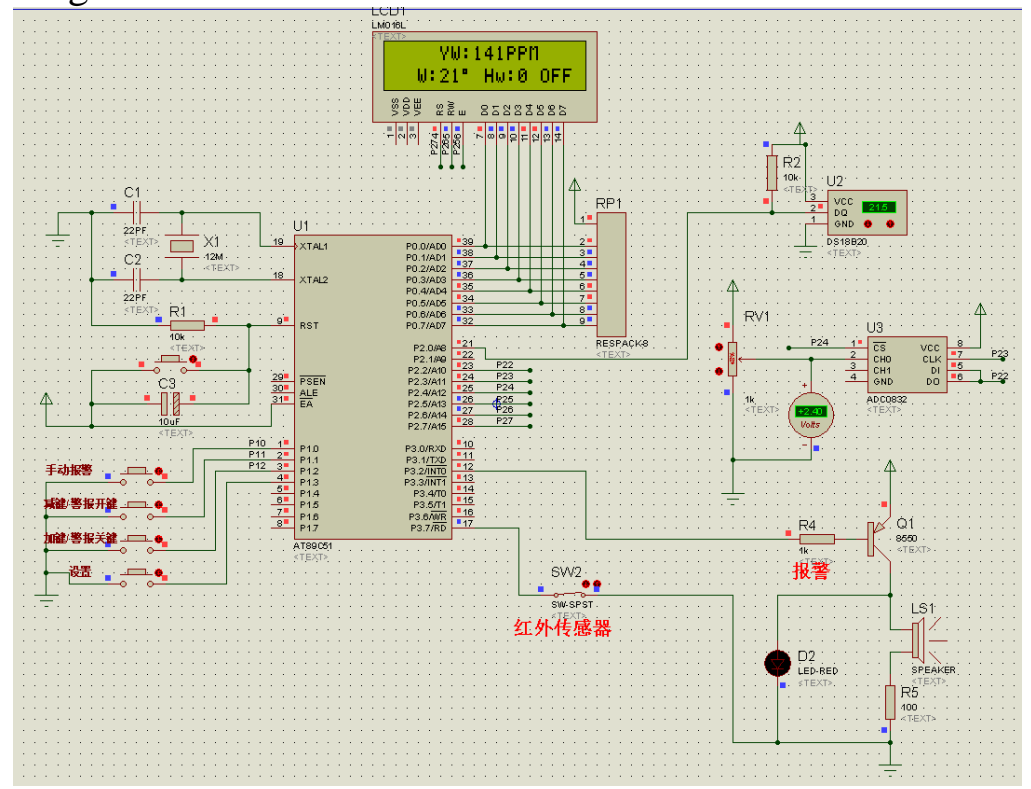

Figure 6 Proteus simulation

\section{Conclusions}

The design consists of two parts: Host and slave microcontroller, The function of detecting and transmitting the user's home smoke and personnel intrusion is realized by Slave microcontroller. The function of detecting and transmitting the user's home smoke and personnel intrusion is realized by host microcontroller. The host realizes the receiving and processing of the signals sent by the slave microcontroller, and monitors the functions of the slave microcontroller and the warning. Through the test, it is found that the system has completed the design requirements, running well and highly practical.

\section{Acknowledgements}

the research fund of Guangxi key laboratory of new energy and building energy saving(No. 15-J-21-12),

\section{References}

[1] Wang Dong. Design of intelligent lighting system based on STM32 and HC-SR501 smart home [J]. Journal of Chongqing University of Technology, 2016,12 (6):43-44.

[2] Wei wei. Detailed explanation of the 51 single-chip microcomputer $\mathrm{C}$ language development and application technical case [M].Beijing chemical industry press [M], 2010.75-78.

[3] Zhou Zhongyan, etc .. Sensing and detection technology [M]. Beijing Institute of Technology Press, 2015.46-49.

[4] Gao Yunhong. Intelligent instrument technology and engineering example design [M]. Beijing University of Aeronautics and Astronautics Press, 2015.150-155.

[5] DU Yu-keng.Design of Wireless Network Based on nRFg05 [J]. Electronic Technology and Software Engineering, 2015,21 (17): 49-51.

[6] Chen Zhong, et al. Design of control system based on STC89C52 microcontroller [M]., Tsinghua University press, 2015.77-81. 